\title{
The effect of repeated laser stimuli to ink-marked skin on skin temperature - recommendations for a safe experimental protocol in humans
}

Victoria J Madden, Mark J Catley, Luzia Grabherr, Francesca Mazzola, Mohammad Shohag, G Lorimer Moseley

Background: Nd:YAP laser is widely used to investigate the nociceptive and pain systems, generating perpetual and laser-evoked neurophysiological responses. A major procedural concern for the use of Nd:YAP laser stimuli in experimental research is the risk of skin damage. The absorption of Nd:YAP laser stimuli is greater in darker skin, or in pale skin that has been darkened with ink, prompting some ethics boards to refuse approval to experimenters wishing to track stimulus location by marking the skin with ink. Some research questions, however, require laser stimuli to be delivered at particular locations or within particular zones, a requirement that is very difficult to achieve if marking the skin is not possible. We thoroughly searched the literature for experimental evidence and protocol recommendations for safe delivery of Nd:YAP laser stimuli over marked skin, but found nothing. Methods: We designed an experimental protocol to define safe parameters for the use of Nd:YAP laser stimuli over skin that has been marked with black dots, and used thermal imagine to assess the safety of the procedure at the forearm and the back. Results: Using thermal imaging and repeated laser stimulation to ink-marked skin, we demonstrated that skin temperature did not increase progressively across the course of the experiment, and that the small change in temperature seen at the forearm was reversed during the rest periods between blocks. Furthermore, no participant experienced skin damage due to the procedure. Conclusion: This protocol offers parameters for safe, confident and effective experimentation using repeated Nd:YAP laser on skin marked with ink, thus paving the way for investigations that depend on it. 
1

2 marked skin on skin temperature -

3 recommendations for a safe experimental

4 protocol in humans

5

6 Authors:

7 Victoria J Madden ${ }^{\mathrm{a}}$, torymadden@gmail.com

8 Mark J Catley ${ }^{a}$, mark.catley@unisa.edu.au

9 Luzia Grabherr ${ }^{a}$, luziagrabherr@gmx.ch

10 Francesca Mazzola ${ }^{\mathrm{a}}$, mazzolavincenzi@alice.it

${ }^{a}$ Sansom Institute for Health Research, University of South Australia, Adelaide, Australia.

${ }^{b}$ Neuroscience Research Australia, Sydney, Australia.

*Address for correspondence: Body in Mind research group, GPO Box 2471, University of South Australia, Adelaide 5001. Email: lorimer.moseley@gmail.com. Tel: +6188302 1416. Fax: N/A. Institutional URL: www.unisa.edu.au

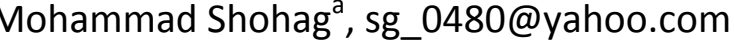

*G Lorimer Moseley ${ }^{\mathrm{a}, \mathrm{b}}$, lorimer.moseley@gmail.com

The effect of repeated laser stimuli to ink- 


\section{Manuscript to be reviewed}

\section{Introduction}

24

Laser stimulation is an important tool for investigating the nociceptive and pain systems, because it allows for the selective activation of nociceptive neurons with a brief, tightly controlled stimulus. Although $\mathrm{CO}_{2}$ lasers have been widely used, the skin discolouration they produce (Lefaucheur et al. 2012) and difficulties with using them in small spaces and at awkward angles have prompted a shift in favour of solid state lasers (e.g. Cruccu et al. 2003). Solid state lasers such as Neodymium Yttrium-Aluminium-Perovskite (Nd:YAP) lasers may be preferable due to their greater flexibility: the machines tend to be less cumbersome, and transmission of the laser through a flexible fibre-optic cable means that these stimuli can be delivered inside an MRI tunnel (Perchet et al. 2008). Many experimental protocols require repeated stimulus application (for example, to measure thresholds, assess acuity, etc.). However, when laser is used for repetitive stimulation there is a risk of progressive temperature increase if the same skin area is heated too often. Progressive increases in skin temperature over the course of a procedure may (a) elevate the risk of skin damage and (b) compromise the reliability of the outcome being studied - for example, perceptual acuity, perceptual intensity or ERP amplitude. This risk may be elevated in people with chronic pain because there are emerging data that suggest spatially defined dysfunction in thermoregulation and cortical stimulus processing in these groups (e.g. Moseley et al. 2012a; Moseley et al. 2012b; Moseley et al. 2012c). To reduce this risk, the location of the stimulus is usually shifted slightly between trials to prevent heating of the same skin area in successive trials. This shifting of location is also thought to reduce sensitisation or habituation to the stimulus (lannetti et al. 2003; Wiech et al. 2010).

This risk of temperature increase and consequent skin damage presents an obvious problem because, to our knowledge, there are no published data outlining the number or frequency of $\mathrm{Nd}$ :YAP laser stimuli that is safe. A further issue is the quandary presented when researchers want to accurately localise stimulation sites: it is generally considered that marking the skin with ink is problematic because darkened skin absorbs the radiant heat more rapidly, exposing it to risk of damage (Leandri et al. 2006). One option would be to mark the skin with white ink. However, pale markings are difficult to see on pale skin, making this approach unsuitable for procedures in which an experimenter must quickly 


\section{Manuscript to be reviewed}

54 localise stimulation sites. Furthermore, marking stimulation sites with a colour that is

55

56

57

58

59

60

61

62

63

64

65

66

67

68

69

70

71

72

73

74

75

76

77

78

79

80

81

82

83

difficult to see makes experimenter fatigue more likely, and could thus compromise accuracy. Using black pens for skin markings is common practice in other experiments that deliver stimuli to human skin and would be a convenient and sensible option for studies that use laser stimuli, if such markings can be used without increasing skin temperature enough to compromise reliability or cause skin damage.

An additional 'safety' check that is commonly used in studies involving painful stimuli is to calibrate the stimulus intensity to individual participants according to pain rating (Mancini et al. 2011; Wager et al. 2006; Weiss et al. 1997). This is a reasonable approach for preventing undue suffering that could be caused by the experimental pain percept. However, pain is influenced by a multitude of factors, including attention (Villemure \& Bushnell 2002), salience (Wiech et al. 2010), emotion (Wiech \& Tracey 2009), task demands (Petrovic et al. 2000) and expectations (Atlas \& Wager 2012). Consequently, stimulus calibration according to pain percept may not be a useful strategy to ensure safety. The finding that people with chronic pain have alterations in perceptual acuity (Wand et al. 2011) suggests that the stimulus energy-percept mismatch may be even more pronounced in people with pain, emphasising that experimental procedures for studying pain must be tested for safety in those with and those without pain.

Here we present data from an experimental protocol in which multiple stimuli were applied to the black-ink-marked skin of 15 people with chronic low back pain and 13 healthy control participants, and thermal imaging was used to evaluate skin temperature before and after stimulation blocks. We quantified the effect of repeated stimulation on skin temperature to ascertain the safety of an inter-stimulus interval and block-rest-block protocol when the locations of stimuli have been marked with black ink.

\section{Methods}

\section{Participants}

We recruited adult volunteers using flyers and word of mouth. Volunteers were not eligible if they had sensation problems, diagnosed peripheral vascular disease, diabetes mellitus, or psychological or neurological problems, or unusual skin conditions (e.g. dermographism) that might compromise skin safety with laser application. Volunteers with skin too dark for 
84

possible erythema to be noticed were also ineligible. Participants had to be pain-free or have chronic back pain, defined as pain between the levels of T12 and S1 (with or without concurrent leg pain)(Merskey \& Bogduk 1994). Volunteers with back pain were excluded if they had concurrent neck or arm pain, were pregnant or less than six months post-partum, or had had spinal surgery. Participants were remunerated at AU\$20/hr for their involvement. All procedures conformed to the Helsinki Declaration and were approved by the institutional ethics committee.

\section{Experimental procedure}

Participants received laser stimuli to the skin of the forearm and the back. Prior to stimulation, the hair on the dorsal forearm and the back was trimmed using clippers, and a template was used to draw two dot-grids onto the skin with a black Artline ${ }^{\circledR} 200$ FINE 0.4 pen. Dots were spaced $4 \mathrm{~mm}$ apart in the proximal-distal (forearm) or medial-lateral (back) direction, and $5 \mathrm{~mm}$ apart in the radial-ulnar (forearm) or cephalo-caudad (back) direction. The forearm grid measured $48 \mathrm{~mm} \times 20 \mathrm{~mm}$; the back grid measured $80 \mathrm{~mm} \times 20 \mathrm{~mm}$ (see Figures 1 and 2).

\section{Stimuli}

Laser stimuli were delivered using an Nd:YAP laser (Deka: Stimul 1340, wavelength $1340 \mathrm{~nm}$, spot diameter measured at $3.5 \mathrm{~mm}$, pulse width $6 \mathrm{~ms})$, which has previously been shown to activate A $\delta$ nociceptors specifically (lannetti et al. 2006). The intensity of laser stimulus for each participant was established, on the basis of an ascending staircase procedure, before testing began. The operator delivered laser stimuli of changing intensity, asking participants to describe what they felt. Once a painful pinprick was elicited, participants rated the pain intensity on a 0-10 numerical rating scale with anchors of "no pain" $(0)$ and "worst pain ever felt" (10). The operator aimed to identify the intensity that consistently elicited pinprick pain of 2-3 out of 10, and this was used for the testing procedure. If a participant reported a diminution or an increase of pain intensity during the procedure, the operator adjusted the laser stimulus intensity accordingly, with a maximum permitted laser intensity of 2.0J. This level was established during piloting as a limit that would prevent skin damage, and thus became an additional exclusion criterion (if a laser stimulus intensity of 2.0J produced less than 2/10 pinprick pain). Participants received stimuli in pairs. Three blocks of 14 stimulus pairs were delivered to the forearm and three blocks of 16 pairs to the back, with at least 30 
115 seconds between pairs and 3-8 minutes between blocks (See Figure 3). The interval

116 between pairs was selected to reduce perceptual habituation, and the break between

117 blocks was chosen to allow participants relief from the sustained concentration required by

118 the perceptual acuity task. Blocks were randomly ordered. Each stimulus was delivered

119 over a dot in the dot-grid. The two stimuli of each pair were delivered $4-44 \mathrm{~mm}$ (at the

120 forearm) or 4-76mm (at the back) apart. This approach was part of a wider plan to evaluate

121 perceptual acuity at each location. It was impossible for any one location to be stimulated

122 twice without a break of at least 120 seconds. The operator monitored skin colour visually,

123 so as to detect any localised erythema that could indicate undue skin heating.

124 Outcome

125 We used infrared thermal imaging (FLIR SC620 camera, FLIR systems, Oregon, USA;

126 sensitivity $<40 \mathrm{mK}$, field of view $=24 \times 18^{\circ}$ ) to record the average skin temperatures within

127 the demarcated zones before and after each stimulation block. This camera produced an

128 image that is colour-coded by infrared radiation. The ThermaCAM Researcher Professional

129 software (version 2.9, FLIR systems, Oregon, USA) allows the user to delineate a certain area

130 and calculate absolute temperature parameters for that area. In this study, the demarcated

131 stimulation zone (forearm grid $48 \mathrm{~mm} \times 20 \mathrm{~mm}$; back grid $80 \mathrm{~mm} \times 20 \mathrm{~mm}$ ) was delineated

132 and the calculation was made as an average for that area. Skin condition was visually

133 monitored throughout the procedure.

134 Statistical Analysis

135 Temperature data were analysed using two analyses of variance (ANOVA): (1) temperatures

136 before and after each block were compared using repeated-measures ANOVA with Time

137 (before/after block) and Site (forearm/back) and Block (1/2/3) as within-subject factors, and

138 Group (patient/healthy) as the between-subject factor, and (2) forearm skin temperatures

139 before each block were compared using repeated-measures ANOVA with Block (1/2/3) as

140 the only factor. Planned comparisons were used to investigate significant effects, and

141 Bonferroni adjustments were applied to correct for multiple comparisons. Alpha was set at

142 0.05. Where the assumption of sphericity was violated, adjusted values are reported, with

143 degrees of freedom also adjusted accordingly. 


\section{Manuscript to be reviewed}

\section{Results}

145 In this experiment, each participant received 84 stimuli at the forearm and 96 stimuli at the

146 back, delivered over a period of $\sim 70$ minutes.

147 We recruited 29 adult volunteers, one of whom was excluded for dermographism. Of the 28

148 adults tested, 15 of them had chronic low back pain (mean age \pm SD $=43 \pm 17$ years; 8

149 female) and 13 were pain-free (mean age $\pm S D=49 \pm 14$ years; 8 female).

150 Overall, skin temperature was greater at the back than at the forearm (main effect of Site, $151 F(1,26)=9.23, p=.005$ ). Skin temperature increased over each block (main effect of Time, $152 F(1,26)=19.45, p<.001)$ and the extent of the increase was greater in the forearm than it 153 was in the back (Time $\times$ Site interaction, $F(1,26)=30.028, p<.001$ ). Comparisons of the Time $154 \times$ Site interaction showed that forearm skin temperature increased over a block $(p<.001$, 155 mean change $\left.\pm \mathrm{SE}=0.40 \pm 0.07^{\circ} \mathrm{C}, 95 \% \mathrm{Cl} 0.27-0.54\right)$ and that back skin temperature did 156 not change over a block ( $p=.098$, mean change $\pm \mathrm{SD}=-0.08 \pm 0.05^{\circ} \mathrm{C}, 95 \% \mathrm{Cl}-0.02-0.18$ ).

157 There was no main effect of Group $(p=0.938)$ or Block $(p=0.626)$ on skin temperature and 158 there were no other significant interactions.

159 Despite the small change in forearm temperature over each block, there was no difference 160 between skin temperatures at the start of each block (no effect of Block, $p=.248$ ),

161 indicating that the rest periods between blocks were sufficient for the forearm skin 162 temperature to return to baseline.

163 None of our participants had localised erythema sufficient to warrant termination of the 164 procedure, none had any visible indications of skin damage on completion of the 165 experiment, and none reported adverse effects afterwards.

166

167

168

169

170

171

172

173

\section{Discussion}

In this study, Nd:YAP laser stimuli were applied to the ink-marked skin of 28 participants without resultant skin damage or lasting heating of the skin. No participant reported skin damage due to the procedure. The thermal imaging data showed no overall increase in skin temperature across the course of the experiment. Skin temperature at the back did not change within blocks. Skin temperature at the forearm increased within each block, but that increase was reversed during the rest periods between blocks such that skin temperatures at the start of each forearm block did not differ. 
174 These results demonstrate that it is possible to use Nd:YAP laser stimuli repeatedly over ink-

175 marked skin without inducing skin damage, provided that strict safety parameters are

176 observed.

177 There were four factors that ensured the safety of this design. The first factor was strict

178 exclusion criteria. We excluded participants with neurological or sensation problems, and

179 we ensured that skin tone was pale enough for the operator to detect any laser-induced

180 change in skin colour. Although Nd:YAP does not produce the same discoloured skin lesions

181 that $\mathrm{CO}_{2}$ laser is known to, whether it produces lesions of deeper layers of the skin is

182 unknown (lannetti et al. 2006). We therefore considered the visual monitoring of skin

183 colour a necessary precaution in this study, and planned to exclude any volunteer in whom

184 this would not be possible.

185 The second factor was the selection of a conservative upper limit to the intensity of laser to

186 be used in the experiment. This limit was based on pilot work in our lab, during which two

187 participants (both males with Fitzpatrick skin type III, 50 and 24 years old) developed minor

188 skin lesions after repeated application of stimuli over $2 \mathrm{~J}$, despite reporting less than 2/10

189 pain at such intensities. Those pilot subjects would not have been excluded on the basis of

190 the other exclusion criteria. We therefore recommend a conservative upper limit to the

191 intensity of laser to be used in an experiment, so as to identify and exclude participants who

192 may be at risk of skin burn during a procedure in which the laser stimulus intensity is

193 determined according to subjective ratings. Our results also indicate that calibration of

194 stimulus intensity according to pain report was not dangerous in this group of participants

195 with and without chronic back pain. Considering that people with chronic pain may display

196 a greater energy-percept mismatch than their healthy counterparts (Wand et al. 2011),

197 there is reason to expect that report-based calibration of stimulus intensity may be unsafe.

198 However, this study did not find evidence of skin damage with the stimuli used, which may

199 be attributable to the 2J safety limit on stimulus energy.

200 The third factor that ensured participants' safety was the use of break periods, which

201 allowed for recovery of skin temperature towards a baseline level between stimulation

202 blocks. The fourth factor that ensured the safety of this design was that the ink markings

203 used in this study were small dots made with a $0.4 \mathrm{~mm}$-diameter pen. As such, the

204 blackened skin area constituted a very small fraction of the area over which the laser

205 stimulus was delivered (mark diameter $0.4 \mathrm{~mm}$; beam diameter $3.5 \mathrm{~mm}$ ), probably resulting

PeerJ reviewing PDF | (2015:09:6973:1:1:NEW 15 Dec 2015) 


\section{Manuscript to be reviewed}

206 in a differential heating effect over the area of surface exposed to the beam. Blackened skin

207 is expected to absorb Nd:YAP laser more superficially, leading to quicker absorption and

208 dissipation of heat, and more selective activation of $A \delta$ fibres than would be expected in un-

209 blackened skin (Leandri et al. 2006). In this study, the rate at which skin temperature

210 recovered from laser stimulation was likely linked to the comparatively small area over

211 which the skin was blackened. Any future work based on this report will need to consider

212 these four factors in order to achieve equivalent safety.

213 Limitations

214 This report provides safe parameters for Nd:YAP laser stimulation using a $3.5 \mathrm{~mm}$ spot

215 diameter. Further work would be required to determine safe parameters for other spot

216 sizes, because the area over which the stimulus is delivered affects the extent of skin

217 heating. If researchers require moment-by-moment information on skin temperature

218 changes, real-time thermal imaging will be necessary. We used imaging before and after

219 blocks, and are therefore unable to provide data on skin temperature between stimuli

220 within a block.

221 A reasonable concern about this report is that we did not personally reassess participants'

222 skin condition in the days following the procedure. It is possible that participants developed

223 delayed signs of skin damage, particularly considering that any strongly absorbent regions of

224 tissue could be subject to greater risk of heat-induced damage due to an unequal heating

225 effect. However, participants were explicitly asked to report any signs of skin damage, and

226 we received no such reports. Furthermore, the intensity limit of $2 \mathrm{~J}$ was established on the

227 basis of pilot testing that showed no skin damage in pilot participants, according to visual

228 assessments made immediately after the procedure and in the following days. We are

229 therefore confident that the parameters presented here did not result in visible skin

230 damage in this group of 28 participants.

\section{Conclusion}

This procedure offers parameters for safe and effective experimentation using Nd:YAP laser and black ink skin markings when delivering stimuli to the back and the forearm of healthy participants and participants with chronic back pain. 
236 Acknowledgements

237 The authors are grateful to Dr Tasha R Stanton for sharing her extensive knowledge about

238 Nd:YAP laser stimuli. Part of this work was presented at the 15th World Congress on Pain 239 (2014).

250

251 References

252 Atlas LY, and Wager TD. 2012. How expectations shape pain. Neuroscience Letters 520:140-

253 148. http://dx.doi.org/10.1016/j.neulet.2012.03.039

254 Cruccu G, Pennisi E, Truini A, lannetti GD, Romaniello A, Le Pera D, De Armas L, Leandri M,

255 Manfredi M, and Valeriani M. 2003. Unmyelinated trigeminal pathways as assessed by laser

256 stimuli in humans. Brain 126:2246-2256. 10.1093/brain/awg227

257 Iannetti GD, Truini A, Romaniello A, Galeotti F, Rizzo C, Manfredi M, and Cruccu G. 2003.

258 Evidence of a Specific Spinal Pathway for the Sense of Warmth in Humans. J Neurophysiol

259 89:562-570. 10.1152/jn.00393.2002

260 Iannetti GD, Zambreanu L, and Tracey I. 2006. Similar nociceptive afferents mediate

261 psychophysical and electrophysiological responses to heat stimulation of glabrous and hairy

262 skin in humans. The Journal of Physiology 577:235-248. 10.1113/jphysiol.2006.115675

263 Leandri M, Saturno M, Spadavecchia L, lannetti GD, Cruccu G, and Truini A. 2006.

264 Measurement of skin temperature after infrared laser stimulation. Clinical Neurophysiology

265 36:207-218. http://dx.doi.org/10.1016/j.neucli.2006.08.004

266 Lefaucheur JP, Ahdab R, Ayache SS, Lefaucheur-Ménard I, Rouie D, Tebbal D, Neves DO, and

267 Ciampi de Andrade D. 2012. Pain-related evoked potentials: A comparative study between

268 electrical stimulation using a concentric planar electrode and laser stimulation using a CO2

269 laser. Neurophysiologie Clinique/Clinical Neurophysiology 42:199-206.

270 http://dx.doi.org/10.1016/j.neucli.2011.12.003

271 Mancini F, Longo MR, lannetti GD, and Haggard P. 2011. A supramodal representation of the

272 body surface. Neuropsychologia 49:1194-1201.

273 http://dx.doi.org/10.1016/j.neuropsychologia.2010.12.040

274 Merskey H, and Bogduk N. 1994. Classification of chronic pain: descriptions of chronic pain 275 syndromes and definitions of pain terms: IASP press Seattle. 
276 Moseley GL, Gallace A, and lannetti GD. 2012a. Spatially defined modulation of skin

277 temperature and hand ownership of both hands in patients with unilateral complex regional

278 pain syndrome. Brain 135:3676-3686. 10.1093/brain/aws297

279 Moseley GL, Gallace A, and Spence C. 2012b. Bodily illusions in health and disease:

280 Physiological and clinical perspectives and the concept of a cortical 'body matrix'.

281 Neuroscience \& Biobehavioral Reviews 36:34-46.

282 http://dx.doi.org/10.1016/j.neubiorev.2011.03.013

283 Moseley GL, Gallagher L, and Gallace A. 2012c. Neglect-like tactile dysfunction in chronic

284 back pain. Neurology 79:327-332. 10.1212/WNL.0b013e318260cba2

285 Perchet C, Godinho F, Mazza S, Frot M, Legrain V, Magnin M, and Garcia-Larrea L. 2008.

286 Evoked potentials to nociceptive stimuli delivered by $\mathrm{CO} 2$ or Nd:YAP lasers. Clinical

287 Neurophysiology 119:2615-2622. http://dx.doi.org/10.1016/j.clinph.2008.06.021

288 Petrovic P, Petersson KM, Ghatan PH, Stone-Elander S, and Ingvar M. 2000. Pain-related

289 cerebral activation is altered by a distracting cognitive task. Pain 85:19-30.

290 http://dx.doi.org/10.1016/S0304-3959(99)00232-8

291 Villemure C, and Bushnell MC. 2002. Cognitive modulation of pain: how do attention and 292 emotion influence pain processing? Pain 95:195-199.

293 Wager TD, Matre D, and Casey KL. 2006. Placebo effects in laser-evoked pain potentials. 294 Brain, Behavior, and Immunity 20:219-230. http://dx.doi.org/10.1016/j.bbi.2006.01.007 295 Wand BM, Parkitny L, O'Connell NE, Luomajoki H, McAuley JH, Thacker M, and Moseley GL. 296 2011. Cortical changes in chronic low back pain: Current state of the art and implications for 297 clinical practice. Manual Therapy 16:15-20. http://dx.doi.org/10.1016/j.math.2010.06.008 298 Weiss T, Kumpf K, Ehrhardt J, Gutberlet I, and Miltner WHR. 1997. A bioadaptive approach 299 for experimental pain research in humans using laser-evoked brain potentials. Neuroscience 300 Letters 227:95-98. http://dx.doi.org/10.1016/S0304-3940(97)00320-0

301 Wiech K, Lin C-s, Brodersen KH, Bingel U, Ploner M, and Tracey I. 2010. Anterior Insula Integrates Information about Salience into Perceptual Decisions about Pain. The Journal of Neuroscience 30:16324-16331. 10.1523/jneurosci.2087-10.2010 and neural mechanisms. Neurolmage 47:987-994. 
1

Figure 1: Dot-grids as drawn onto forearm (a) and back (b). Two dot-grids are pictured at each site: the second was used for tactile stimulation (data not presented here).




2

Diagram depicting experimental procedure and main results. CLBP: people with chronic low back pain. 


\section{Procedure}

Forearm stimulation zone
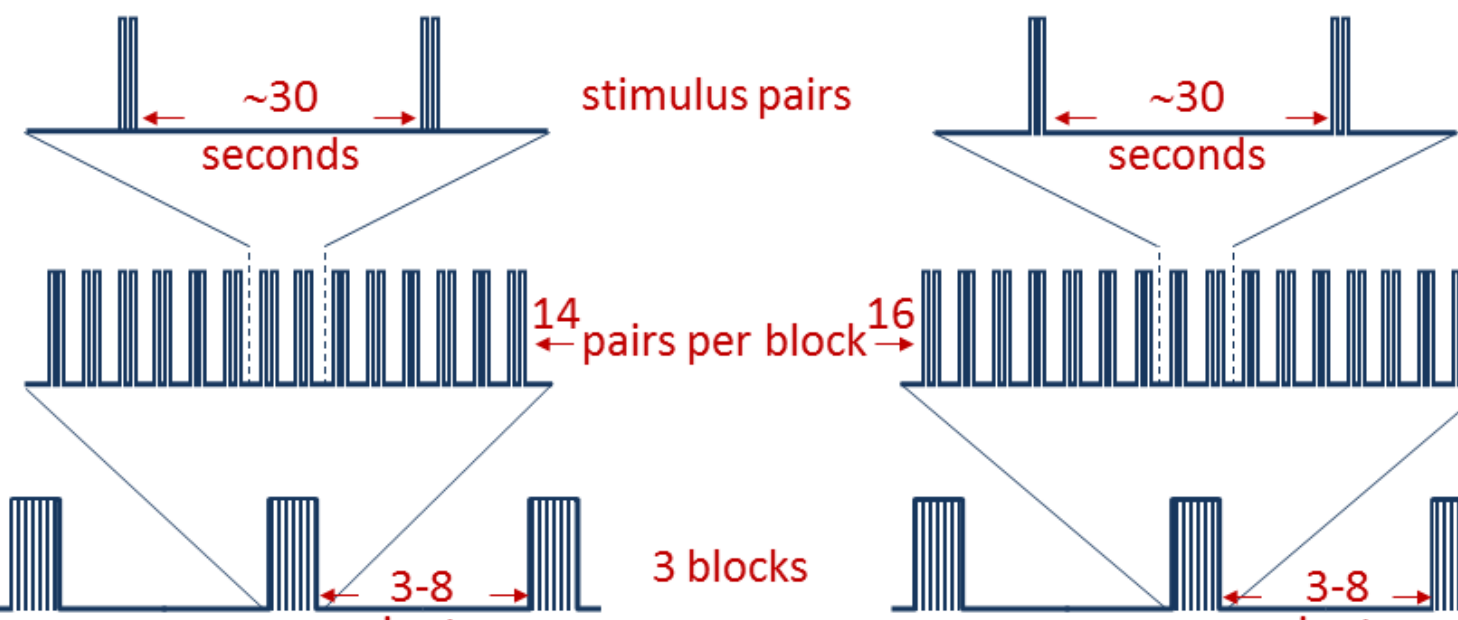

minutes
Back stimulation zone

14 pairs per block $\stackrel{16}{-}$

3 blocks

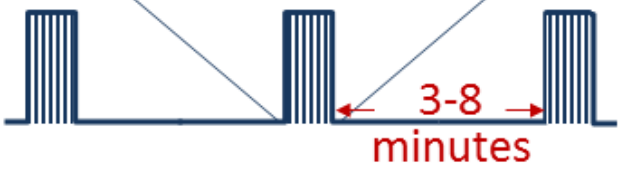

\section{Results}

Average skin temperature in stimulation zones over time

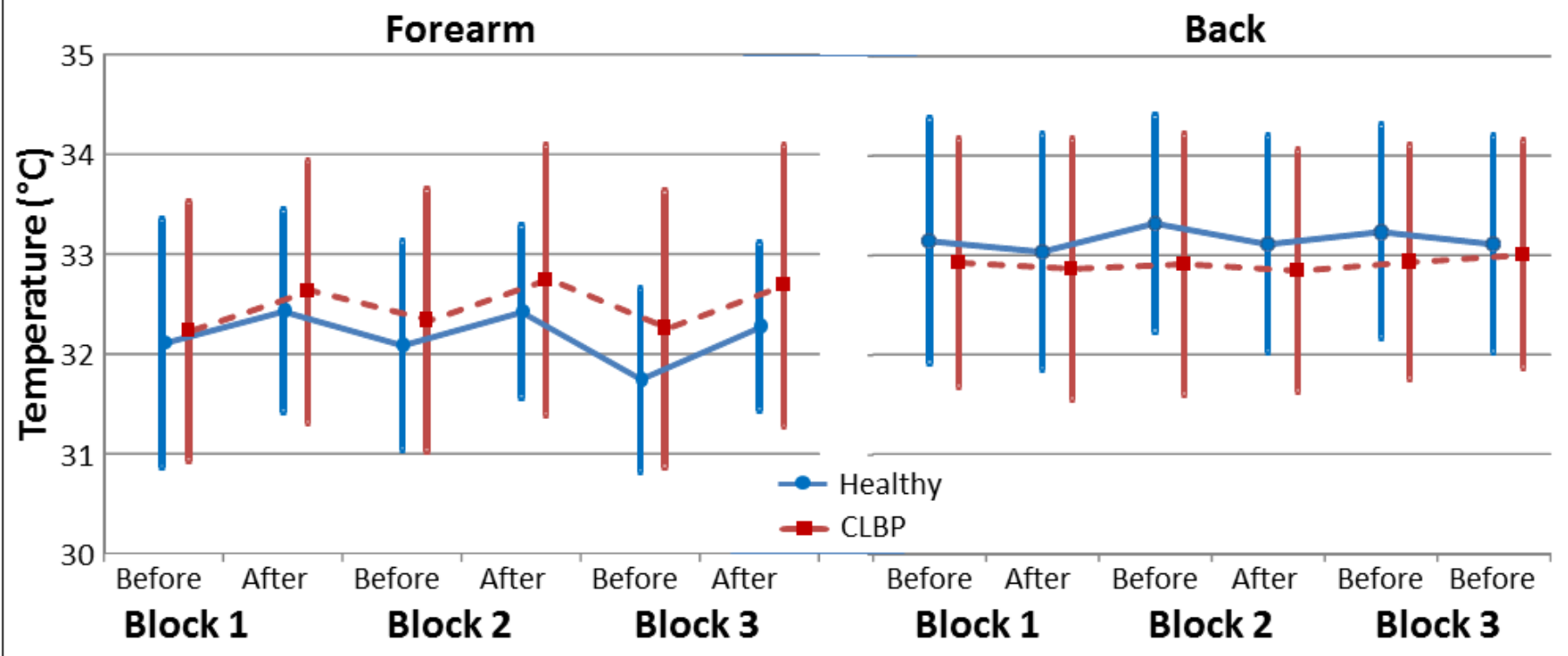

